\title{
Optical excitations of Si by time-dependent density-functional theory based on the exact-exchange Kohn-Sham band structure
}

\author{
Yong-Hoon Kim ${ }^{1}$, Martin Städele ${ }^{2}$, and Andreas Görling ${ }^{1}$ \\ ${ }^{1}$ Lehrstuhl für Theoretische Chemie, Technische Universität München, D-85748 Garching, Germany and \\ ${ }^{2}$ Infineon Technologies AG, CPR ND, Otto-Hahn-Ring 6, D-81730 Munich, Germany
}

\begin{abstract}
We calculate the imaginary part of the frequency-dependent dielectric function of bulk silicon by applying time-dependent density-functional theory based on the exact-exchange (EXX) Kohn-Sham (KS) band structure and the adiabatic local-density approximation (ALDA) kernel. The position of the $E_{2}$ absorption peak calculated with the EXX band structure at the independent-particle level is in excellent agreement with experiments, which demonstrates the good quality of EXX 'KS quasiparticles'. The excitonic $E_{1}$ peak that is missing at the independent-particle level remains absent if two-particle interaction effects are taken into account within the time-dependent LDA, demonstrating the incapability of the ALDA kernel to describe excitonic effects.
\end{abstract}

PACS numbers: 71.15.Mb,71.35.Cc,78.20.Ci

\section{INTRODUCTION}

While the Kohn-Sham (KS) density-functional theory (DFT) [1, 2, 3] has become the dominant method of firstprinciples investigations of energetic properties of solids, its scope in the treatment of electronic excitations has been rather limited due to deficiencies of the conventional local density approximation (LDA) and its semilocal extensions. One well-known case is the band gaps calculated within the LDA which are substantially smaller than experimental values [2]. Significant advancement has been made during the past decades in devising improved exchange-correlation functionals starting from the LDA [3], but these semilocal approximations still fail to correct the so-called 'band-gap problem'. Thus for the accurate treatment of band structures one at present usually leaves the domain of DFT and employs a manybody method based on the GW approximation (GWA) to quasiparticle energies [4, 5].

For the case of optical excitations in which bound exciton states can play a significant role, it is further required to go beyond effective one-particle approaches. In principle, this can be done within the time-dependent (TD) DFT [6, 7] or by solving the Bethe-Salpeter equation (BSE) for two-body Green's functions [8]. However, the lack of a proper exchange-correlation functional again remains as the hindrance to the TDDFT route, and the GWA-BSE currently represents the state-of-the-art method for such purpose [9, 10, 11].

In this regard recent numerical realization of the exactexchange (EXX) KS method for solids 12, 13, 14] and molecules 115, 16, 17, 18, 19, 20 provides an interesting opportunity: According to theoretical and numerical evidence concerning the physical meaningfulness of the KS potential and KS eigenvalues [21, 22, 23], realistic band structures for semiconductors and eigenvalue spectra of molecules obtained within the EXX suggests a promising basis for the study of electronic excited states within DFT. In this work, we consider this point further by calculating the absorption spectrum of bulk silicon within
TDDFT based on the EXX band structure and the adiabatic LDA (ALDA) exchange-correlation kernel. Silicon here serves as a representative semiconductor system, whose first absorption peak, the $E_{1}$ peak, is known to be strongly excitonic in its character. The large excitonic contributions to the $E_{1}$ peak has been proposed theoretically and experimentally, and a semiempirical calculation confirming this point has been performed two decades ago [8]. However, truly first-principles calculations appeared only recently based on the GWA-BSE [9, 10, 11. A different approach to investigate optical spectra within a DFT framework was recently explored in Ref. 24, where the domain of conventional DFT was left and timedependent current DFT was invoked. As a first step towards an accurate computation of optical spectra within TDDFT, we will examine the performance of the EXXTDLDA approach in this paper.

\section{THEORY}

To obtain the absorption spectrum of solids, we compute the imaginary part of the frequency-dependent macroscopic dielectric function $\epsilon(\omega)=\epsilon_{1}(\omega)+i \epsilon_{2}(\omega)$ defined as 25, 26]

$$
\epsilon(\omega)=\left[\left.\lim _{q \rightarrow 0} \epsilon^{-1}\left(\mathbf{q}+\mathbf{G}, \mathbf{q}+\mathbf{G}^{\prime} ; \omega\right)\right|_{\mathbf{G}=\mathbf{G}^{\prime}=0}\right]^{-1}
$$

where

$$
\begin{aligned}
\epsilon^{-1}\left(\mathbf{q}+\mathbf{G}, \mathbf{q}+\mathbf{G}^{\prime} ; \omega\right) & =\delta_{\mathbf{G}, \mathbf{G}^{\prime}}+F_{H}(\mathbf{q}+\mathbf{G}) \\
& \times \chi\left(\mathbf{q}+\mathbf{G}, \mathbf{q}+\mathbf{G}^{\prime} ; \omega\right),
\end{aligned}
$$

with $F_{H}(\mathbf{q}+\mathbf{G}) \equiv 4 \pi /|\mathbf{q}+\mathbf{G}|^{2}$ (Hartree atomic units are used throughout the paper. The symbols $\mathbf{q}+\mathbf{G}$ refer to plane waves with wave vectors $\mathbf{q}+\mathbf{G}, e^{i(\mathbf{q}+\mathbf{G}) \cdot \mathbf{r}}$, i.e., all formulas are given in the plane wave representation). The linear response function $\chi$ that describes the full response of the first-order density change $\delta n$ for the given external 
perturbation $\delta v_{\text {ext }}$,

$\delta n(\mathbf{q}+\mathbf{G} ; \omega)=\sum_{\mathbf{G}^{\prime}} \chi\left(\mathbf{q}+\mathbf{G}, \mathbf{q}+\mathbf{G}^{\prime} ; \omega\right) \delta v_{e x t}\left(\mathbf{q}+\mathbf{G}^{\prime} ; \omega\right)$,

can be computed either via the BSE or TDDFT. Within TDDFT the linear response function $\chi$ is given in matrix representation as [6, ,7]

$\chi(\mathbf{q} ; \omega)=\left[1-\chi_{0}(\mathbf{q} ; \omega)\left\{F_{H}(\mathbf{q})+F_{x c}(\mathbf{q} ; \omega)\right\}\right]^{-1} \chi_{0}(\mathbf{q} ; \omega)$.

where $\chi_{0}$ is the noninteracting KS linear response matrix. It is given in terms of the occupied and unoccupied $\mathrm{KS}$ orbitals $\phi_{v \mathbf{k}}$ and $\phi_{c \mathbf{k}}$ and their eigenvalues $\epsilon_{v \mathbf{k}}$ and $\epsilon_{c \mathbf{k}}$ through

$$
\begin{aligned}
& \chi_{0}\left(\mathbf{q}+\mathbf{G}, \mathbf{q}+\mathbf{G}^{\prime} ; \omega\right)=\frac{2}{N \Omega} \\
& \times \sum_{v, c, \mathbf{k}}\left[\frac{\left\langle v, \mathbf{k}\left|e^{-i(\mathbf{q}+\mathbf{G}) \cdot \mathbf{r}}\right| c, \mathbf{k}+\mathbf{q}\right\rangle\left\langle c, \mathbf{k}+\mathbf{q}\left|e^{i\left(\mathbf{q}+\mathbf{G}^{\prime}\right) \cdot \mathbf{r}^{\prime}}\right| v, \mathbf{k}\right\rangle}{\epsilon_{v \mathbf{k}}-\epsilon_{c(\mathbf{k}+\mathbf{q})}+\omega+i \delta}\right. \\
& \left.+\frac{\left\langle c, \mathbf{k}\left|e^{-i(\mathbf{q}+\mathbf{G}) \cdot \mathbf{r}}\right| v, \mathbf{k}+\mathbf{q}\right\rangle\left\langle v, \mathbf{k}+\mathbf{q}\left|e^{i\left(\mathbf{q}+\mathbf{G}^{\prime}\right) \cdot \mathbf{r}^{\prime}}\right| c, \mathbf{k}\right\rangle}{\epsilon_{v \mathbf{k}}-\epsilon_{c(\mathbf{k}+\mathbf{q})}-\omega-i \delta}\right]
\end{aligned}
$$

where $N \Omega$ is the crystal volume. In Eq. (4) $F_{x c}$ denotes the exchange-correlation kernel, the frequency-dependent functional derivative of the exchange-correlation potential.

Thus, the macroscopic dielectric function $\epsilon$ can be computed by first calculating $\epsilon^{-1}$ in the reciprocal-space using Eqs. (11) and (5), and next taking the inverse of the $q \rightarrow 0$ limit of its $\mathbf{G}=\mathbf{G}^{\prime}=0$ element [Eq. (11)]. However, the ALDA kernel $F_{x c}^{A L D A}=\delta^{2} E_{x c}^{L D A} /\left(\delta n \delta n^{\prime}\right)$ is local in real space and frequency-independent, which results in a reciprocal-space representation for $F_{x c}$ that is independent of $q$ and $\omega, F_{x c}^{A L D A}\left(\mathbf{q}+\mathbf{G}, \mathbf{q}+\mathbf{G}^{\prime} ; \omega\right)=$ $F_{x c}^{A L D A}\left(\mathbf{G}-\mathbf{G}^{\prime}\right)$. This incorrect $q \rightarrow 0$ behavior of the ALDA kernel then simplifies the calculation of $\epsilon(\omega)$ : Writing $\chi, F_{H}$, and $F_{x c}$ (from now on the superscript "ALDA" on the kernel is suppressed for notational simplicity) for $q \rightarrow 0$ in block form in terms of the "head" $\left[\mathbf{G}=\mathbf{G}^{\prime}=0(00)\right]$, "wings" $\left[\mathbf{G}=0 ; \mathbf{G}^{\prime} \neq 0(01)\right.$ and $\left.\mathbf{G} \neq 0 ; \mathbf{G}^{\prime}=0(10)\right]$, and the "body" $\left[\mathbf{G} \neq 0 ; \mathbf{G}^{\prime} \neq 0\right.$ (11)] as

$$
\begin{aligned}
\chi_{0}(q ; \omega) & =Q\left(\begin{array}{cc}
\chi_{0}^{00}(\omega) & \chi_{0}^{01}(\omega) \\
\chi_{0}^{10}(\omega) & \chi_{0}^{11}(\omega)
\end{array}\right) Q ; \\
F_{H}(q) & =Q^{-1}\left(\begin{array}{cc}
4 \pi & 0 \\
0 & F_{H}^{11}
\end{array}\right) Q^{-1} ; \\
F_{x c}(q) & =Q^{-1}\left(\begin{array}{cc}
0 & 0 \\
0 & F_{x c}^{11}
\end{array}\right) Q^{-1}
\end{aligned}
$$

where $Q^{00}=q ; Q^{01}=Q^{10}=0 ; Q^{11}=\delta_{\mathbf{G}, \mathbf{G}^{\prime}}$, and performing matrix multiplications explicitly (with qsingularities treated properly at each stage) yields a $3 \times 3$ tensor [25, 26],

$$
\begin{aligned}
\epsilon(\omega)=1- & 4 \pi \chi_{0}^{00}(\omega)-4 \pi \chi_{0}^{01}(\omega)\left(F_{H}^{11}+F_{x c}^{11}\right) \\
& \times\left[1-\chi_{0}^{11}(\omega)\left(F_{H}^{11}+F_{x c}^{11}\right)\right]^{-1} \chi_{0}^{10}(\omega),
\end{aligned}
$$

with which matrix multiplications including head and wings with explicit q-dependence are avoided. Eq. (7) simply represents the independent-particle-level absorption spectrum if $F_{H}^{11}$ and $F_{x c}^{11}$ are ignored altogether, while it is reverted to the so-called RPA dielectric function formula if only $F_{x c}^{11}$ is neglected. For cubic systems, such as Si considered here, the macroscopic dielectric tensor is diagonal.

\section{COMPUTATIONAL METHODS}

For the calculation of the dielectric function, one needs wavefunctions $\phi_{i \mathbf{k}}$ and eigenvalues $\epsilon_{i \mathbf{k}}$ at the chosen set of $\mathbf{k}$-points [See Eq. (5)]. To generate these, we first performed the LDA and EXX self-consistent groundstate calculations at the experimental lattice constant, $a=5.43 \AA$ for $\mathrm{Si}$, and obtained the KS potential $v_{K S}$ which serves as the only input to the TDDFT calculation of optical spectra. The EXX calculation was carried out using ten special-k points, orbital kinetic energy cutoffs of $12.5 \mathrm{Ha}$, and nonlocal pseudopotentials in the separable form of Kleinman-Bylander [27]. We refer the reader to Ref. 14 for further details of EXX calculations. In addition, because the ALDA kernel $F_{x c}^{A L D A}$ depends only on the density, we constructed it at the end of the ground-state calculations when fully converged densities were available.

With the converged $v_{K S}^{E X X}, v_{K S}^{L D A}$ and $F_{x c}^{A L D A}$ in hand, we moved on to the computation of the dielectric function and first solved the KS equations once again at a much larger number of $\mathbf{k}$-points but with the same energy cutoff 12.5 Ha to generate $\chi_{0}$. The calculation of the head and wings of $\chi_{0}$ needs special care since the $q \rightarrow 0$ limit of $\left\langle i, \mathbf{k}\left|e^{-i \mathbf{q} \cdot \mathbf{r}}\right| j, \mathbf{k}+\mathbf{q}\right\rangle$ should be handled properly. Applying perturbation theory one obtains the following equation 26],

$$
\lim _{q \rightarrow 0}\left\langle i, \mathbf{k}\left|e^{-i \mathbf{q} \cdot \mathbf{r}}\right| j, \mathbf{k}+\mathbf{q}\right\rangle=\mathbf{q} \cdot \frac{\left\langle i, \mathbf{k}\left|\mathbf{p}+\left[V_{N L}, i \mathbf{r}\right]\right| j, \mathbf{k}\right\rangle}{\epsilon_{j, \mathbf{k}}-\epsilon_{i, \mathbf{k}}}
$$

which implies that, due to the extra powers of the energy denominator that appear in the head $\chi_{0}^{00}(\hat{\mathbf{q}} ; \omega)$, computation parameters for the convergence are more or less determined by those necessary for the head element. We use the kinetic energy cutoff $7 \mathrm{Ha}$ and 10 conduction bands for $\chi_{0}$ which was sufficient to reach the convergence of $\epsilon_{2}(\omega)$.

For the k-point sampling, we chose to adopt a regular uniform grid, with which one could evaluate $\lim _{q \rightarrow 0}\left\langle i, \mathbf{k}\left|e^{-i \mathbf{q} \cdot \mathbf{r}}\right| j, \mathbf{k}+\mathbf{q}\right\rangle$ by a finite difference approach without resorting to the analytic replacement of Eq. (8). 
In this case one had to pay the price of performing three additional diagonalizations at $\mathbf{k}+q \mathbf{e}_{\alpha}(\alpha=x, y, z)$ for each k-point. Therefore, in this paper we report results obtained by treating the $q \rightarrow 0$ limit according to Eq. (8) with ignoring the nonlocal pseudopotential contributions due to the commutator $\left[\widehat{V}_{N L}, i \mathbf{r}\right]$ term. In test calculations with a small number of k-points we found that the neglect of the nonlocal pseudopotential contributions has little effect on the positions of absorption peaks while the peak heights are somewhat reduced. We slightly shift the uniform $\mathbf{k}$-mesh in order to break any present symmetry thus achieve a more effect $\mathbf{k}$-point sampling [11. After checking the convergence behavior by increasing the number of k-points and changing the shifting vector, we used a shifted $17 \times 17 \times 17$ regular grid which gives the convergence of $\epsilon_{2}$ up to the frequency range of at least $6 \mathrm{eV}$. The use of a shifted uniform $\mathrm{k}$-mesh results in slightly non-identical diagonal elements of the dielectric tensor whose differences have been also used to check the convergence with respect to the number of $\mathbf{k}$-points.

\section{RESULTS AND DISCUSSIONS}

We first consider the absorption spectrum calculated at the LDA and EXX independent particle levels. In Fig. 1, LDA and EXX absorption spectra are shown together with experimental [28] and GWA [9, 10] curves. Note that the two GWA results are rather different due to operational ambiguities resulting from the post-DFT nature of the GWA [5], while the LDA and EXX which strictly reside in the KS DFT scheme do not suffer from such problems. Within the LDA, the absorption spectra is shifted to the lower frequency region by about $1 \mathrm{eV}$ due to its well-known underestimation of bands gaps. In addition, the $E_{1}$ peak height is significantly underestimated, while that of the $E_{2}$ peak is much overestimated. In contrast, the EXX absorption spectrum shows a much better overall agreement reflecting realistic descriptions of the band structure within the EXX [14]: Positions of the absorption edge and the $E_{2}$ peak are in excellent agreement with the experiment, and the $E_{2}$ peak position is even slightly better than the GWA one. Also the height of the $E_{2}$ peak is smaller than the LDA value and is much closer to the experimental data. One noticeable disagreement is the absence of the $E_{1}$ peak as in the LDA spectrum. This, however, does not reflect the deficiency of the EXX approach, but the excitonic nature of the $E_{1}$ peak [9, 10, 11] which cannot be described at the independent particle level as can be also seen in the GWA curves.

We feel that the excellent features of the EXX absorption spectrum warrant more discussions. Perturbation theory along the adiabatic connection shows that KS eigenvalue differences are well-defined approximations of excitation energies 21]. Indeed Umrigar et al. recently found for several atoms that quasi-exact KS eigenvalue differences are in surprisingly good agreement with ex- perimental excitations [22]. In addition, applying the quasi-exact KS potential to the calculation of the quantum defects for the $\mathrm{Ne}$ atom, Al-Sharif et al. argued that the KS potential of a neutral atom (with $N$ electrons) corresponds to the Green-function "potential" of its daughter cation ( $N-1$ electrons), or equivalently the KS equation for $N$ electrons corresponds to the Dyson equation where the reference ground state is chosen with $N-1$ electrons [23]. The appealing description of excitations by KS eigenvalue differences, in contrast to the treatment of ionizations and electron affinities in the many-body (GWA) approach, has not drawn much attention due to the lack of realistic exchange-correlation approximations which are suitable for practical calculations. Exchange-correlation potentials derived from the LDA and other semilocal exchange-correlation energy functionals do not cancel Coulomb self-interactions, decay exponentially rather than as $-1 / r$ for localized systems, and inherently lack the term that results from the functional derivative of the pair-correlation function [29]. The self-interaction-free EXX method corrects such problems and provides accurate KS potentials and eigenvalues. Then, we propose that the good absorption spectrum from such EXX method represents further evidence for the $N$-electron excitations picture within the KS scheme.

Now we consider the effects of the inclusion of two-particle interactions via TDLDA on top of the independent-particle EXX result. The Hartree-only or RPA (EXX+TDH) and full TDLDA $(\mathrm{EXX}+\mathrm{TDH}+\mathrm{ALDA})$ spectra are shown together with the EXX independent-particle spectrum in Fig. 2. Granted the overall good feature of the EXX spectrum, the most interesting question is that whether the ALDA can generate the excitonic $E_{1}$ peak. Fig. 2 shows that this is not the case. TDH alone suppresses the EXX spectrum and shifts the $E_{2}$ peak to a slightly higher frequency region, while adding the ALDA counteracts such effects and more or less restores the EXX independent-particle spectrum. In Fig. 2 we also display the TDLDA absorption spectrum based on the LDA band structure (LDA+TDH+ALDA) to show that the LDA independent-particle result (see Fig. 田) is again insignificantly changed by TDLDA effects.

\section{CONCLUSIONS}

In this work, by calculating the frequency-dependent dielectric function of bulk $\mathrm{Si}$ we studied the quality of EXX one-particle band structure and the description of excitonic effects within the TDLDA. We showed that, at the EXX independent-particle level, the position of the $E_{2}$ peak is in good agreement with experimental data and is even slightly better than that obtained in the GWA approach. We argued that this is because the EXX approach, unlike procedures employing the LDA and semilocal density-functionals, yields realistic KS orbitals 
and eigenvalues which accurately model electronic excitations at a fixed particle number. Employing TDDFT within the ALDA on top of the EXX band structure however results in only a minor modification of the EXX spectrum and fails to produce the excitonic $E_{1}$ peak. Given the fact that proper inclusion of excitonic effects is mandatory for the complete description of optical excitations, devising a TDDFT scheme that overcomes the deficiency of the ALDA kernel will be an interesting task. Work along this line using the nonadiabatic and nonlocal EXX kernel is in progress [30].

\section{Acknowledgments}

We thank Professor W. Domcke for his support including computational resources. This work was supported by the Deutsche Forschungsgemeinschaft and the Fonds der Chemischen Industrie. Y.-H. Kim also acknowledges receipt of a Humboldt Foundation award.
[1] W. Kohn, Rev. Mod. Phys. 71, 1253 (1999)

[2] R. O. Jones and O. Gunnarsson, Rev. Mod. Phys. 61, 689 (1989).

[3] W. Koch and M. C. Holthausen, A Chemist's Guide to Density Functional Theory (Wiley-VCH, New York, 2000), and references therein.

[4] M. S. Hybertsen and S. G. Louie, Phys. Rev. Lett. 55, 1418 (1985); Phys. Rev. B 34, 5390 (1986).

[5] W. G. Aulbur, L. Jönsson, and J. W. Wilkins, Solid State Physics, vol. 54 (Academic, New York, 2000).

[6] E. K. U. Gross, J. F. Dobson, and M. Petersilka, in Density Functional Theory II, Springer Series in Topics in Current Chemistry, Vol. 181, ed. R.F. Nalewajski (Springer, Heidelberg, 1996), p. 81.

[7] M. E. Casida, in Recent Advances in Density Functional Methods, Part I, ed. D.P. Chong (World Scientific, Singapore, 1995), p. 155.

[8] W. Hanke and L. J. Sham, Phys. Rev. Lett. 43, 387 (1979); Phys. Rev. B 21, 4656 (1980).

[9] S. Albrecht, L. Reining, R. Del Sole, and G. Onida, Phys. Rev. Lett. 80, 4510 (1998); 83, 3971 (1999).

[10] L. X. Benedict, E. L. Shirley, and R. B. Bohn, Phys. Rev. Lett. 80, 4514 (1998); L. X. Benedict and E. L. Shirley, Phys. Rev. B 59, 5441 (1999).

[11] M. Rohlfing and S. G. Louie, Phys. Rev. Lett. 81, 2312 (1998); Phys. Rev. B 62, 4927 (2000).

[12] D. M. Bylander and L. Kleinman, Phys. Rev. Lett. 74, 3660 (1995); Phys. Rev. B 52, 14566 (1995); 54, 7891 (1996); 55, 9432 (1997).

[13] A. Görling, Phys. Rev. B 53, 7024 (1996); (E) 59, 10370 (1999).

[14] M. Städele, J. A. Majewski, P. Vogl, and A. Görling, Phys. Rev. Lett. 79, 2089 (1997); M. Städele, M.
Moukara, J. A. Majewski, P. Vogl, and A. Görling, Phys. Rev. B 59, 10031 (1999).

[15] T. Grabo and E. K. U. Gross, Int. J. Quantum. Chem. 64, 95 (1997).

[16] Y.-H. Kim, M. Städele, and R. M. Martin, Phys. Rev. A 60, 3633 (1999).

[17] A. Görling, Phys. Rev. Lett. 83, 5459 (1999).

[18] S. Ivanov, S. Hirata, and R. J. Bartlett, Phys. Rev. Lett 83, 5455 (1999).

[19] L. Veseth, J. Chem. Phys. 114, 8789 (2001).

[20] F. Della Salla and A. Görling, J. Chem. Phys. 115, 5718 (2001).

[21] A. Görling, Phys. Rev. A 54, 3912 (1996).

[22] C. J. Umrigar, A. Savin, and X. Gonze, in Electronic Density Functional Theory: Recent Progress and New Directions, edited by J. F. Dobson, G. Vignale, and M. P. Das (Plenum, New York, 1998).

[23] A. I. Al-Sharif, R. Resta, and C. Umrigar, Phys. Rev. A 57, 2466 (1998).

[24] P. L. de Boeij, F. Kootstra, J. A. Berger, R. van Leeuwen, and J. G. Snijders, J. Chem. Phys. 115, 1995 (2001).

[25] R. M. Pick, M. H. Cohen, and R. M. Martin, Phys. Rev. B 1, 910 (1970).

[26] M. S. Hybertsen and S. G. Louie, Phys. Rev. B 35, 5585 (1987).

[27] L. Kleinman and D. M. Bylander, Phys. Rev. Lett. 48, 1425 (1982).

[28] C. M. Herzinger, B. Johs, W. A. McGahan, and A. Woollam, J. Appl. Phys. 83, 3323 (1998).

[29] O. V. Gritsenko, R. van Leeuwen, E. J. Baerends, J. Chem. Phys. 101, 8955 (1994).

[30] Y.-H. Kim and A. Görling, in preparation. 


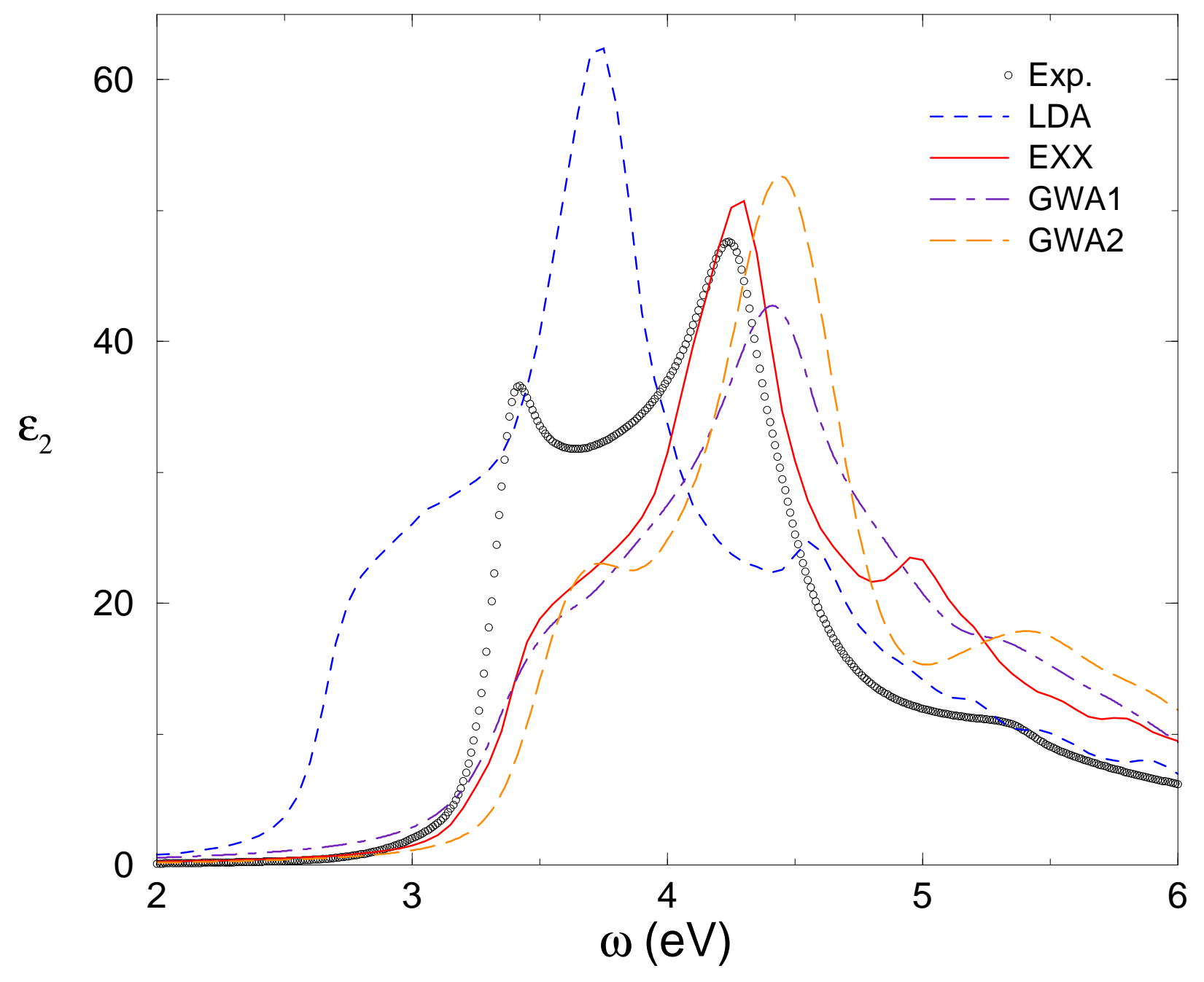

FIG. 1: Absorption spectrum of bulk silicon at the one-particle LDA and EXX levels. Experimental curve is from Ref. 28 . Two GWA results (GWA1 from Ref. 10 and GWA2 from Ref. 9) are also shown for comparison. 


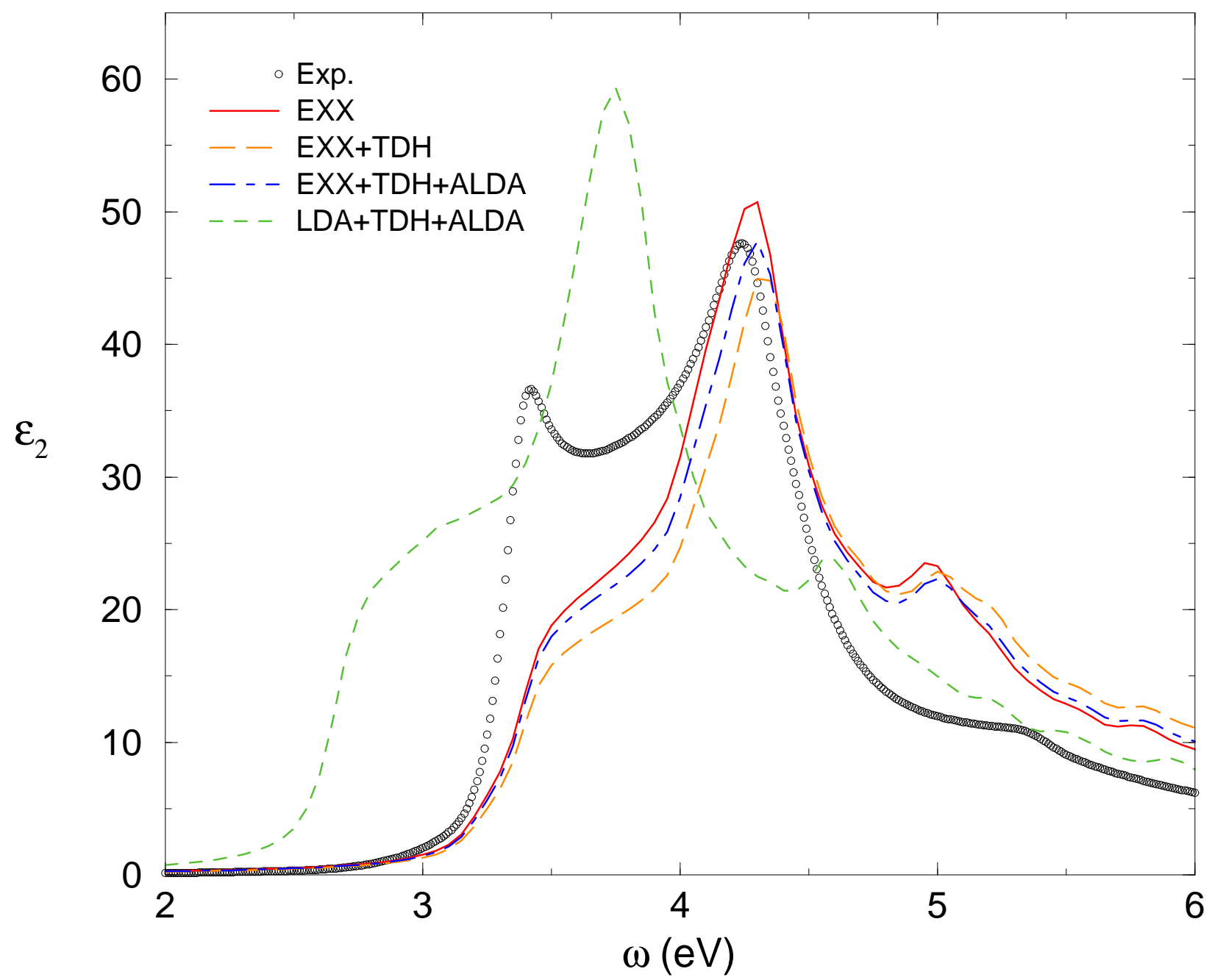

FIG. 2: Absorption spectrum of bulk silicon at the EXX, EXX+TDH, EXX+TDH+ALDA, and LDA+TDH+ALDA levels. Experimental data are from Ref. 28. 